\title{
Evaluation of a Hear-Through Device
}

\author{
Anders Kalsgaard Møller, Pablo Faundez Hoffmann, Flemming Christensen, \\ and Dorte Hammershøi
}

Aalborg University, Department of Electronic Systems, Aalborg, Denmark

$\{a k m, p f h, f c, d h\} @ e s . a a u . d k$

\begin{abstract}
A solution for recreating the natural sound reception of the open ear while wearing earphones exists. It is achieved by mounting microphones on the outside of earphones, and simultaneously record and playback the sound. The solution could potentially be used for augmented audio applications. Due to practical limitations, such as the size of the earphones and microphones, it is not possible to record the sound in the ideal position. This paper presents work on finding the best position of a microphone with the purpose of reproducing as much of the spatial information as possible. The results from measurements with an artificial ear show that all spatial informations can be reproduced up to around 5-6 kHz. Above this frequency deviations in the reproduced sound, compared to the natural, is introduced. A method to evaluate how the deviations in the reproduced sound affect the listening experience is also proposed.
\end{abstract}

Keywords: augmented reality, augmented audio, virtual reality, binaural recordings, hear-through.

\section{Introduction}

In augmented audio applications natural sounds are mixed with virtual sounds. The virtual sound could potentially be played through earphones but when wearing earphones the earphones will to some degree block the ear canals and dim the sounds from the listener's surroundings. By mounting microphones on the outside of the earphones and simultaneously record and playback the sound, the natural sound reception of the open ear can be recovered.

A device which can simultaneously record and reproduce the sound surrounding the user is presented in this paper and will be referred to as a hear-through device. The hear-through device consist of a pair of earphones AKG328 Blue and two MEMS microphones(Analog Device, ADMP504). The earphones and the microphone is connected to an Arduino Due board with a codec shield used to handle the recording, playback, and equalizing.

The recordings done by the hear-through device is based on the principles of the binaural recording technique. According to Møller [1] the complete auditory experience can be preserved by recording and reproducing the sound pressure at each eardrum exactly as it were. Hammershøi and Møller [2] concluded that recordings at the blocked entrance to the ear canal also include all the spatial information and is therefore a more suitable place for making binaural recordings.

C. Stephanidis (Ed.): HCII 2014 Posters, Part I, CCIS 434, pp. 440-445, 2014.

(C) Springer International Publishing Switzerland 2014 
Even though the earphones and microphones available today are quite small it is not possible to record the sound exactly at the blocked entrance to the ear canal but instead in a position sticking a bit out from the ideal position.

In 3] a method is presented for measuring the transfer functions for the ideal position, at the blocked entrance to the ear canal, and the position of an in-ear device respectively. The in-ear device consisted of an earphone with a build-in microphone. Later [4] continued the work with several different types of earphones.

The principles of a hear-through device have previously been described and tested [5] and potential applications have been described in [6]. The work included the design of a hear-through device and a small-scale usability evaluation. The usability evaluation revealed some issues to the listener experience such as the sound of the listeners own voice and annoying noise levels. The general impression of the audio quality was however good.

The hear-through device offers new options as a tool for HCI-applications where the real sound environment is combined with computer-generated sound. This could for instance be used for a museum guide where the user receive information about the attraction when moving close to it or in other ways offer options for interaction with the attraction. The augmented audio could also be combined with other modalities like vision to create an augmented reality that immerse the user into an intriguing environment.

Before adding a virtual sound it is however important to be able to reproduce the natural sound environment or at least be able to measure to what degree this can be accomplished. If the natural sound can't be reproduced correct it might compromise the experience of the augmented reality.

In this paper the work on a hear-through device is described. The work include finding the optimal position of the microphones and earphones for reproducing the spatial information and it include an subjective evaluation of the auditory experience created using the hear-through.

\section{Method}

This section describe the method used to find the optimal position of the microphone on the earphone.

\subsection{Setup}

The measurements to find the optimal position of the earphone were performed in an anechoic room. The measurement system used in this method consisted of 25 loudspeakers distributed on a half sphere with 22.5 degree angular difference between the loudspeaker positions. The sphere was attached to a baffle with a hole in the middle with room for an ear to stick out (either an artificial ear or a human ear). The loudspeakers suspension construction was wrapped in acoustically absorbing material in order to minimize reflections from the setup. In Figure 1 the setup is presented. 

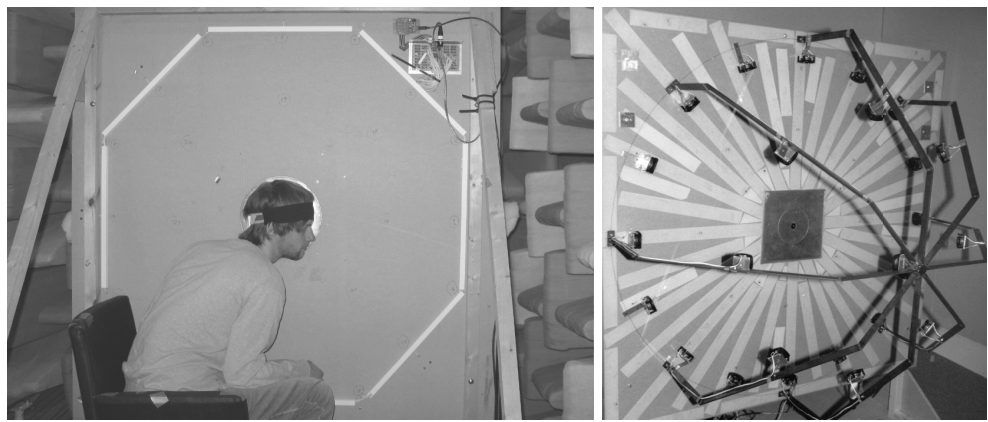

Fig. 1. The front and back of the baffle used in the setup (Source: [3]

\subsection{Measurements}

A custom-built measurement system was used to play Maximum Length Sequence noise through each of the loudspeakers, one at a time, and compute the impulse responses.

One measurement was conducted with a MEMS microphone(Analog Devices, ADMP504) attached to the blocked ear canal of the artificial ear. Another eight measurements were conducted with the same microphone attached in different positions on the AKG328 Blue earphones. Figure 2 shows the microphone positioned on the earphone and on the blocked entrance to the ear canal.

Another series of measurement were conducted using five human subjects. The subjects were placed in a chair with their ear sticking out of the baffle. For each subject, a measurement with the microphone attached to the blocked ear canal and three measurements with the microphone positioned at selected positions on the earphone, were conducted.

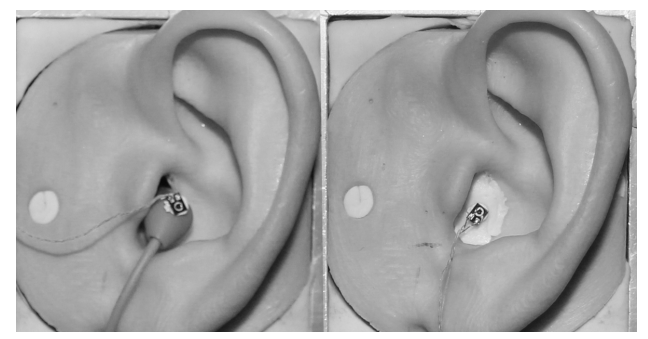

Fig. 2. To the left a microphone placed on the earphone in the artificial ear. To the right a microphone placed at the blocked entrance to the ear canal. 


\section{$3 \quad$ Results}

Figure 3 shows the amplitude response of the transfer function with the artificial ear. The amplitude response for each direction is very similar up to around 5-6 $\mathrm{kHz}$ where they start to separate. This was the best result observed out of the eight positions measured with the artificial ear. The position of the microphone on the earphone is the same as on Figure 2.
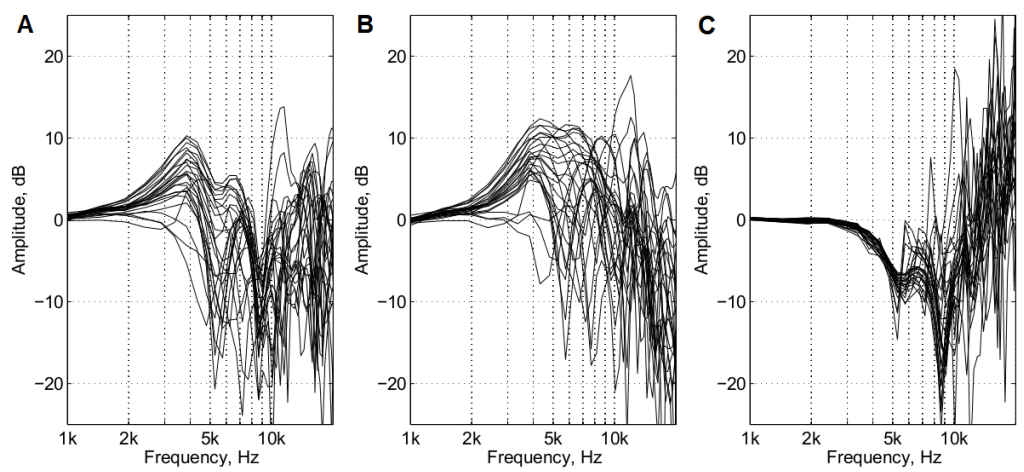

Fig. 3. Amplitude response of transfer function measurement conducted with the artificial ear. (A) Microphone placed at the blocked entrance to the ear canal. (B) Microphone placed on a selected position on the earphone. (C) Difference between blocked entrance to the ear canal and earphone.
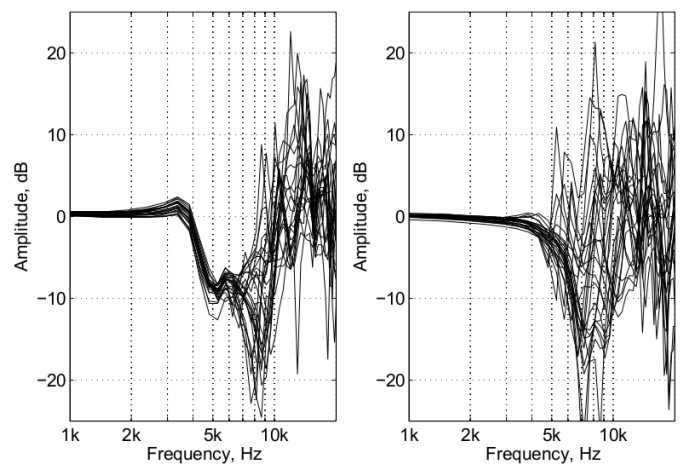

Fig. 4. Amplitude response of transfer function measurement conducted with human ears. Each plot represents the difference between a measurement at the blocked entrance to the ear canal and a position on the earphone. The two plots represent two different subjects with the microphone placed at two different positions. 
In the measurements with the human ears the position of the microphone on the earphones is also compared with the position at the blocked entrance to the ear canal. Figure 4 presents two selected subjects with the microphone placed at a different position for each subject. On the plot to the left the amplitude response for each direction are very similar up to around $5-7 \mathrm{kHz}$ where they starts to separate. This was the best case out of all the measurements with the human subjects. On the plot to the right the amplitude response for each direction start to separate around $4 \mathrm{kHz}$. This was the tendency in most of the measurements.

\section{Subjective Evaluation}

The purpose of the subjective evaluation is to determine how the listening experience is affected by the hear-through device.

The evaluation involves groups of three subjects who are instructed to solve a puzzle in a joint effort. The task is chosen because it encourage the subjects to move around and to communicate with each other. Each group is given the task three times, once wearing the hear-through device, once with the natural condition and once with an occluded condition (with the ears blocked with earphones). The subjects will always start with the natural condition. After completing the puzzle together in the natural condition the subjects continue with either the occluded condition or the hear-through condition. The order of these two conditions is balanced. After the second and third session the subjects are asked to answer a questionnaire with six questions. In each question the subjects are asked to rate the session they just completed compared to the natural condition. The subjects are instructed to answer the questions on continuous scales. The questions address the following topics: (1) Communication (ability to understand what the other subjects said). (2) Sound of the subjects own voice (If their own voice sounded natural). (3) Localization. (4) The annoyance due to noise. (5) The naturalness of the sound from the surroundings. (6) The overall sound quality.

After completing the three sessions the subjects are interviewed to elucidate other issues besides the ones being measured through the questionnaire and to further describe the issues addressed in the questionnaire.

\section{Discussion}

The results achieved from measuring the position of the microphone placed on the AKG328 Blue earphones is a small improvement from the measurement conducted in [3] and at least comparable to the measurements conducted in [4]. The AKG328 earphones can therefore be considered a good choice for the hear-through device. The earphones are quite small and allow the microphone to be placed very close to the ideal position at the blocked entrance to the ear canal. The results from measuring the ears of the human subjects showed that some variation from ear to ear is added. This could both be due to the different 
geometry of the pinna and due to the different fit in the ear canal. The optimal position of the microphone could not be decided based on the small group of subjects and further measurements will have to be conducted.

The hear-through's representation of the spatial information deviate from the natural acoustics of the listeners surroundings. The deviations are however frequency dependent, and are minor in the frequency range of human speech. It remains uncertain to which degree the listening experience is affected by this, if at all. This is evaluated in the ongoing experiment.

\section{References}

1. Møller, H.: Fundamentals of Binaural Technology. Applied Acoustics 36, 171-218 (1992)

2. Hammershøi, D., Møller, H.: Sound transmission to and within the human ear canal. J. Acoust. Soc. Am. 100 (1996)

3. Christensen, F., Hoffmann, P.F., Hammershøi, D.: Measuring directional characterstics of in-ear recording devices. Audio Engineering Society Convention 134 (2013)

4. Hoffmann, P.F., Christensen, F., Hammershøi, D.: Quantitative assessment of spatial sound distortion by the semi-ideal recording point of a hear-through device. In: Proceedings of Meeting on Acoustics, vol. 19 (2013)

5. Riikonen, V., Tikander, M., Karjalainen, M.: An augmented Reality Audio Mixer and Equalizer. Audio Engineering Society Convention 124 (2008)

6. Lokki, T., Nironen, H., Vesa, S., Savioja, L., Härmä, A., Karjalainen, M.: Application Scenarios of Wearable and Mobile Augmented Reality Audio. Audio Engineering Society Convention 116 (2004) 